\title{
Role of Prophylactic Noninvasive Ventilation in Patients at High Risk of Extubation Failure
}

\author{
Lalit Singh
}

\author{
Indian Journal of Critical Care Medicine (2020): 10.5005/jp-journals-10071-23692
}

Prolonged mechanical ventilation (MV) has serious side effects and complications. Thus, one opts for an early extubation after correcting the causes and stabilizing the patient. ${ }^{1}$ Extubation is commonly uneventful especially in an odds ratio (OR) but in intensive care unit (ICU) it is often associated with respiratory failure development post post extubation which may very often require reintubation. The rule of thumb about reintubation risk cannot be applied to all patients as the pathophysiology of extubation failure is poorly understood.

An average of $15 \%$ patients may need reintubation among which $25-30 \%$ are at high risk. ${ }^{1}$ The high-risk patients of extubation failure include preterm infants, age $\geq 65$ years, any respiratory disease, cardiac disease. ${ }^{2}$ A major cause of weaning failure is acute respiratory failure (ARF) due to respiratory muscle fatigue or increased work of breathing due to decreased pulmonary compliance or increased resistance. Other causes include inadequate cough, airway obstruction, excess secretions, neurologic impairment. An important factor responsible for mortality in ICU patients after extubation is ARF. Noninvasive ventilation (NIV) is used for both management and prevention of post-extubation respiratory failure.

After extubation, oxygenation can be improved by three methods available: conventional oxygen therapy, high-flow oxygen therapy, and NIV. Respiratory support is most commonly provided by conventional oxygen therapy. However, in recent years, highflow oxygen therapy (HFOT) and NIV are being used increasingly. These methods are speculated to prevent extubation failure by promoting alveolar recruitment, preventing alveolar collapse, and reducing the work of breathing. Noninvasive ventilation (NIV) causes an increase in the intrathoracic pressure preventing alveolar collapse, improving oxygenation, and reducing the workload of the heart. It also prevents complications of invasive MV. ${ }^{3}$ The protocol of using prophylactic NIV can involve the immediate application of NIV within 1 hour of extubation for a duration of 8-24 hours depending upon the improvement in the respiratory parameters, such as, respiratory rate, $\mathrm{pH}$, partial pressures of oxygen, and carbon dioxide. The prophylactic use of NIV has been adopted for reducing the rates of reintubation, duration of $\mathrm{MV}$, and improving the overall prognosis of patients at high risk of post-extubation failure., ${ }^{1,5}$

The International Consensus Conference in intensive care medicine in 2001 suggested that NIV is a promising therapy to prevent respiratory failure after weaning. ${ }^{6}$ It can ameliorate some of the pathophysiologic derangements that occur following extubation. It has been used as an adjunct to weaning or as a part of early extubation approach.

Since the need for reintubation has been associated with significantly poor outcomes in terms of prolongation of the ICU
Department of Respiratory and Critical Care Medicine, Shri Ram Murti Smarak Institute of Medical Sciences, Bareilly, Uttar Pradesh, India

Corresponding Author: Lalit Singh, Department of Respiratory and Critical Care Medicine, Shri Ram Murti Smarak Institute of Medical Sciences, Bareilly, Uttar Pradesh, India, Phone: +91 9415134949, e-mail: lalitsinghdr@gmail.com

How to cite this article: Singh L. Role of Prophylactic Noninvasive Ventilation in Patients at High Risk of Extubation Failure. Indian J Crit Care Med 2020;24(12):1158-1160.

Source of support: Nil

Conflict of interest: None

stay, hospital stay, use of MV and its associated complications like pneumonia and lung damage, the requirement of tracheotomy, and financial implications, two studies have used different methods for preventing it; among which the prophylactic use of NIV has been found to be is successful. ${ }^{4,5,7}$ The successful use of prophylactic NIV has been most pronounced in high-risk patients of extubation failure. ${ }^{8}$ Postextubation respiratory failure and reintubation prevention by using NIV is supported by weak evidence. NIV can decrease reintubation rates was concluded in two meta-analyses, but these studies had both high risk (only 35\%) as well as the general population., ${ }^{9,10}$ NIV compared to with conventional oxygen therapy in ICU patients at high risk of reintubation was found to be more effective. ${ }^{4}$ However, a metaanalysis conducted in 2014 with 1,382 patients found that the use of NIV as a preemptive measure after extubation or after respiratory failure which developed post-extubation was not beneficial either in reducing mortality or intubation rate. ${ }^{10}$

Liu et al. ${ }^{3}$ found that prophylactic NIV brings about a significant reduction in the atelectasis $(\mathrm{OR}=0.43, p=0.02)$ and rate of reintubation ( $\mathrm{OR}=0.33, p=0.02)$. The reduction in atelectasis rate is an additional advantage since the development of atelectasis is associated with further complications, such as, pneumonia and atelectrauma. ${ }^{11}$ One of the previous review studies backed up the improved outcomes of the use of prophylactic NIV in major abdominal surgeries. ${ }^{12}$ This was even seen on the patients undergoing cardiothoracic surgeries where prophylactic NIV reduced the rate of reintubation. ${ }^{13}$ In a landmark study by Thille et al. ${ }^{1}$ which compared the use of prophylactic NIV in 150 high-risk extubated patients with 83 control extubated patients in an ICU setting, there was a significant reduction in reintubation in the study group ( $15 \%$ vs $28 \%, p=0.02$ ). The study is important since it included high-risk patients with cardiac disease and respiratory disease, and found that prophylactic NIV was an independent predictor of extubation success. Their findings were backed up by

\footnotetext{
(0) The Author(s). 2020 Open Access This article is distributed under the terms of the Creative Commons Attribution 4.0 International License (https://creativecommons. org/licenses/by-nc/4.0/), which permits unrestricted use, distribution, and non-commercial reproduction in any medium, provided you give appropriate credit to the original author(s) and the source, provide a link to the Creative Commons license, and indicate if changes were made. The Creative Commons Public Domain Dedication waiver (http://creativecommons.org/publicdomain/zero/1.0/) applies to the data made available in this article, unless otherwise stated.
} 
two studies prior to before it which showed a significant reduction in the reintubation from 24 to $8 \%, p=0.027$ and from 39 to $5 \%, p$ $=0.016$, respectively. ${ }^{4,5}$

In an RCT, Ferrer et al. ${ }^{14}$ concluded that early use of NIV averted respiratory failure after extubation and decreased mortality in high-risk patients. Another RCT of 648 patients at high risk of extubation failure concluded that the use of high-flow nasal oxygen with NIV immediately after extubation significantly decreased the risk of reintubation. ${ }^{15}$ Nava et al. in a randomized controlled trial studied the use of prophylactic NIV in patients at high risk of extubation failure following at least 48 hours of invasive ventilation and extubation after a successful spontaneous breath trial. They observed that the rate of reintubation was significantly lower in the prophylactic NIV group (8\%) compared to with the control group (24\%) and NIV was associated with significantly lower ICU mortality and ICU length of stay. ${ }^{4}$ Ferrer et al. ${ }^{14}$ applied NIV immediately post-extubation, with age $>65$ years and APACHE II score $>12$ at extubation as a factor for high risk of extubation failure and observed that post-extubation respiratory failure was lower in the NIV group (16\% vs $33 \%$ ) but there was no difference in the rate of re-intubation and ICU mortality and 90-days mortality was significantly lower in NIV group. Ferrer et al. in their RCT, used prophylactic NIV in patients with underlying chronic respiratory illness and hypercapnia at extubation. Prophylactic NIV was associated with lower respiratory failure at 48 hours post-extubation and significantly lower 90 -day mortality. ${ }^{16}$

In this issue, Ghosh et al. ${ }^{17}$ studied outcomes of prophylactic NIV at extubation after a planned extubation, in patients at a high risk of extubation failure. They observed extubation success in $88.2 \%$ of patients at 72 hours. Higher age, longer duration of invasive ventilation, and higher SOFA score at extubation were the factors associated with extubation failure. They also observed organ failure and higher cumulative fluid balance in the first 72 hours post-extubation in the extubation failure group. Ghosh et al. ${ }^{17}$ and Upadya et al. ${ }^{18}$ observed in their studies that higher cumulative fluid balance at extubation was an independent risk factor for extubation failure in patients after planned extubation.

Among the newer modalities, Ali et al. ${ }^{19}$ studied the use of nasal high-frequency oscillatory ventilation (NHFOV) as a prophylactic NIV or "rescue mode of NIV" after extubation. The NHFOV is a "noninvasive ventilation mode that applies an oscillatory pressure waveform to the airways using a nasal interface". The results were promising which showed a decreased requirement of reintubation. It is suggested that NHFOW may be a feasible modality that in is being used as prophylactic NIV following extubation for the prevention of apnea and reintubation. But still, the practical applications need randomized controlled trials to lay down the indications and guidelines for its use in other populations. Prophylactic NIV has emerged as an promising modality and its practical use is welcome. Its efficacy has been seen in diverse age groups ranging from pediatric to adults to elderly.

Appropriate patient selection, i.e., those who are at high risk of extubation failure is important, because it is not useful in low-risk patients, but also that its use may be detrimental in some patients.

\section{References}

1. Thille AW, Boissier F, Ben-Ghezala H, Razazi K, Mekontso-Dessap A, Brun-Buisson C, et al. Easily identified at-risk patients for extubation failure may benefit from non-invasive ventilation: a prospective before-after study. Crit Care 2016;20(1):48. DOI: 10.1186/s13054-0161228-2.

2. Thille AW, Boissier F, Ben Ghezala H, Razazi K, Mekontso-Dessap A, Brun-Buisson C. Risk factors for and prediction by caregivers of extubation failure in ICU patients: a prospective study. Crit Care Med 2015;43(3):613-620. DOI: 10.1097/CCM.0000000000000748.

3. Liu Q, Shan M, Liu J, Cui L, Lan C. Prophylactic noninvasive ventilation versus conventional care in patients after cardiac surgery. J Surg Res 2020;246:384-394. DOI: https://doi.org/10.1016/j.jss.2019.09.008.

4. Nava S, Gregoretti C, Fanfulla F, Squadrone E, Grassi M, Carlucci A, et al. Noninvasive ventilation to prevent respiratory failure after extubation in high-risk patients. Crit Care Med 2005;33(11):2465-2470. DOI: 10.1097/01.CCM.0000186416.44752.72.

5. Ornico SR, Lobo SM, Sanches HS, Deberaldini M, Tófoli LT, Vidal $A M$, et al. Noninvasive ventilation immediately after extubation improves weaning outcome after acute respiratory failure: a randomized controlled trial. Crit Care 2013;17(2):R39. DOI: 10.1186/ cc12549.

6. American Thoracic Society, European Respiratory Society, European Society of Intensive Care Medicine. Societe de Reanimation de LANGUE FRANCCAISE. International consensus conferences in intensive care medicine: noninvasive positive pressure ventilation in acute respiratory failure. Am J Respir Crit Care Med 2001;163(1):283291. DOI: 10.1164/ajrccm.163.1.ats1000.

7. Ferrer M, Sellarés J, Valencia M, Carrillo A, Gonzalez G, Badia JR, et al. Noninvasive ventilation after extubation in hypercapnic patients with chronic respiratory disorders: randomised controlled trial. Lancet 2009;374(9695):1082-1088. DOI: 10.1016/S0140-6736(09)61038-2.

8. Su CL, Chiang LL, Yang SH, Lin HI, Cheng KC, Huang YC, et al. Preventive use of non-invasive ventilation after extubation: a prospective, multicenter randomized controlled trial. Respir Care 2012;57(2):204-210.

9. Glossop AJ, Shephard N, Bryden DC, Mills GH. Non-invasive ventilation for weaning, avoiding reintubation after extubation and in the postoperative period: a meta-analysis. $\mathrm{Br} J$ Anaesth 2012;109(3):305-314. DOI: 10.1093/bja/aes270.

10. Lin $\mathrm{C}, \mathrm{Yu} \mathrm{H}, \mathrm{Fan} \mathrm{H}, \mathrm{Li}$ Z. The efficacy of non-invasive ventilation in managing postextubation respiratory failure: a meta-analysis. Heart Lung 2014;43(2):99-104. DOI: 10.1016/j.hrtIng.2014.01.002.

11. Badenes R, Lozano A, Belda FJ. Postoperative pulmonary dysfunction and mechanical ventilation in cardiac surgery. Crit Care Res Pract 2015;2015:420513. DOI: 10.1155/2015/420513.

12. Chiumello D, Chevallard G, Gregoretti C. Non-invasive ventilation in postoperative patients: a systematic review. Intensive Care Med 2011;37(6):918-929. DOI: 10.1007/s00134-011-2210-8.

13. Olper L, Corbetta D, Cabrini L, Landoni G, Zangrillo A. Effects of non-invasive ventilation on reintubation rate: a systematic review and meta-analysis of randomised studies of patients undergoing cardiothoracic surgery. Crit Care Resusc 2013;15:220-227.

14. Ferrer M, Valencia M, Nicolas JM, Bernadich O, Badia JR, Torres A. Early Noninvasive ventilation averts extubation failure in patients at risk: a randomized trial. Am J Respir Crit Care Med 2006;173(2):164-170. DOI: 10.1164/rccm.200505-7180C.

15. Thille AW, Muller G, Gacouin A, Coudroy R, Decavèle M, Sonneville $R$, et al. Effect of postextubation high flow nasal oxygen with noninvasive ventilation vs high flow nasal oxygen alone on reintubation among patients at high risk of extubation failure. JAMA 2019;322(15):1465-1475. DOI: 10.1001/jama.2019.14901.

16. Ghosh S, Chawla A, Jhalani R, Salhotra R, Arora G, Nagar S, et al. Outcome of prophylactic non-invasive ventilation following planned extubation in high- risk patients: 2 year prospective observational study from a general intensive care unit. Indian J Crit Care Med 2020;24(12):1185-1192.

17. Ghosh S, Chawla A, Mishra K, Jhalani R, Salhotra R, Singh A. Cumulative fluid balance and outcome of extubation: a prospective observational study from a general intensive care unit. Indian J Crit Care Med 2018;22(11):767-772. DOI: 10.4103/ijccm.IJCCM_216_18. 
18. Upadya A, Tilluckdharry L, Muralidharan V, Amoateng-Adjepong $Y$, Manthous CA. Fluid balance and weaning outcomes. Intensive Care Med 2005;31(12):1643-1647. DOI: 10.1007/s00134-005-2801-3.
19. Ali YAH, Seshia MM, Ali E, Alvaro R. Noninvasive high-frequency oscillatory ventilation: a retrospective chart review. Am J Perinatol 2020. Online ahead of print. DOI: 10.1055/s-0040-1718738. 